\title{
Optimization Acceleration Integral Method Based on Power Spectrum Estimation
}

\author{
Ruijie Guo ${ }^{12, *}$, Shengbo Ye1 and Yicai Ji ${ }^{1,2}$ \\ ${ }^{1}$ Key Laboratory of Electromagnetic Radiation and Sensing Technology, Chinese Academy of Sciences, Beijing 100190, China \\ ${ }^{2}$ University of Chinese Academy of Sciences, Beijing 100049, China
}

\begin{abstract}
Due to the excellent performance of the frequency domain integration method, it is widely used for acceleration integral calculations. However, the frequency-domain integration needs to select the effective integration frequency band to achieve its optimal integration performance. This paper proposes the method with power spectrum density (PSD) estimation to realize the optimization integral of the acceleration signal. By analysing the power spectrum density of the acceleration signal, the optimal low frequency cut-off frequency is obtained. Combined with frequency domain integration algorithm, it can effectively remove low-frequency noise and improve integral accuracy. Then, the novel algorithm tested by an experiment platform with a vibration bench. Experiment results show that this algorithm can adaptively select the low-frequency cut-off frequency and realize frequency domain integration optimization and the integration error is controlled within $\pm 0.2 \mathrm{~mm}$.
\end{abstract}

\section{INTRODUCTION}

Time domain integration and frequency domain integration are two commonly used integration methods for acceleration integration. However, the presence of DC components in the acquisition signal, temperature drift, unstable sensor low-frequency performance, and ambient environment interference will all can generate trending items in the signal ${ }^{[1-3]}$. The trend items become more obvious when integrating to speed and displacement, which causes the signal to have a baseline drift, more than this, the displacement signal obtained by the integral will be seriously distorted. Therefore, the effective removal of low-frequency noise in the acceleration signal and correction of the signal baseline after integration has become an important subject for researchers to study.

The article ${ }^{[4]}$ proposed to control the integral trend term error by high-pass filtering the signal before and after integration, however, when the frequency of the main component of the signal is low, the filtering is not sufficient, and the phase of each frequency component is easily distorted ${ }^{[5]}$. Wang Jianfeng proposed a method that performing an integration in the time domain first and then performing an integration in the frequency domain in $2010^{[6]}$. This method both can reduce the cumulative error of the time domain integration and reduce the low-frequency truncation error of the frequency domain integration, however, it also has the dis-advantages of both methods. By establishing the trend term control equation, the article ${ }^{[7-8]}$ solved the inverse problem to obtain system characteristics, derived the frequency response function of the acceleration input and displacement output, and then put forward a new filter.The algorithm comprehensively considered the effective control of the integral error while avoiding the loss of low-frequency information as much as possible, and has a wide range of application .However, the calculation process of the new filter is more complicated. When the target frequency is low, the filter transition band becomes shorter, which causes oscillation in the frequency domain ${ }^{[9]}$.

In this paper, we proposed the algorithm with power spectrum density (PSD) estimation to select the cut-off frequency of the frequency integration. Then, the method can obtain the optimization integration results of the acceleration signal.

\section{PRINCIPLE OF FREQUENCY DOMAIN INTEGRATION}

The trapezoidal formula and the Simpson formula are usually used to integrate the measurement signal in the time domain to obtain the displacement signal. Trend terms are usually eliminated by least-square fitting. However, the trend items generated during the integration process cannot be completely removed, and residual small errors will be cumulatively amplified, which will have a great impact on the accuracy of the integration.

The frequency-domain integration method is simple in principle and usually provides good integration results. This method not only can effectively avoid the cumulative amplification effect of time-domain integration on tiny error signals, but also can easily select the cut-off frequency, which will realize the

\footnotetext{
* Corresponding author: guoruijie15@mails.ucas.edu.cn
} 
function of the band-pass filter. Due to its high stability and accuracy, it has become the most widely used method. Firstly, convert the acceleration signal from the time domain to the frequency domain, then use the nature of the forward and inverse Fourier transform:

$$
F\left[\int_{-\infty}^{t} f(t) d t\right]=\frac{1}{j k} F[f(t)]
$$

Then calculate directly in the frequency domain with the sine and cosine integral interchange relationship. After suppress or remove low-frequency components from the signal, then convert the signal to the time domain, so as to achieve the purpose of correcting the baseline. Assuming that the acceleration signal is $a(n)$, its signal in the frequency domain is:

$$
A(k)=\sum_{k=0}^{N-1} a(n) e^{-j 2 \pi k \frac{n}{N}}
$$

Velocity $v(n)$ is obtained by one integral of acceleration, and displacement $s(n)$ can be obtained by quadratic integral. They can be respectively expressed as:

$$
\begin{aligned}
& v(n)=\frac{A}{j k}=\sum_{k=0}^{N-1} \frac{1}{j 2 \pi k \Delta f} H(k) a_{n} e^{-j 2 \pi k \frac{n}{N}} \\
& s(n)=-\frac{A}{k^{2}}=\sum_{k=0}^{N-1} \frac{1}{-(2 \pi k \Delta f)^{2}} H(k) a_{n} e^{-j 2 \pi k \frac{n}{N}}
\end{aligned}
$$

Among them,

$$
H(k)=\left\{\begin{array}{cc}
1 & f_{d} \leq k f \leq f_{u} \\
0 & \text { others }
\end{array}\right.
$$

where $\Delta f$ is frequency resolution, fd and fu are the lower limit and the upper limit of cut-off frequency respectively, $\mathrm{N}$ is the number of data points, $\mathrm{k}$ is the frequency corresponding to the Fourier component. It can be seen from equation (3), frequency domain integration has amplifying effect on low-frequency frequencies, if the low-frequency cut-off frequency is not selected correctly, low frequency noise will be present in the signal, which will directly affect the accuracy of the integration.

The commonly used low-frequency cut-off frequency selection method is based on empirical selection or comparison through a large number of attempts. So there is some obvious defects:

(1) Artificially select of the low-frequency cut-off frequency is needed, which cannot completely eliminate the problem of the trend item, or eliminate the useful signal, so that the integral result will have a large error.

(2) It is not possible to determine which lowfrequency cut-off frequency can minimize the error in the actual signal processing and achieve the best processing effect.

In order to improve the frequency domain integration performance, this paper proposes an acceleration integral method based on PSD estimation. According to the characteristics of the acceleration power spectrum, this method adaptively selects the cut-off frequency and removes trend items as much as possible.

\section{INTEGRATION METHOD BASED ON PSD}

\subsection{Power Spectrum Estimation}

The PSD estimation can obtain the power spectral density of the signal, which is used to estimate the frequency components of the signal. Common PSD estimation methods can be divided into non-parametric PSD estimation and parameter PSD estimation. Different methods have different effects for different types of signals. This paper uses Welch power spectrum estimation to process acceleration signal.

The Welch method belongs to the non-parametric spectral estimation method, which is characterized by two aspects: (1). It allows subsequences $a_{i}(n)$ to overlap each other;(2) It can be windowed for each subsequence, which can generate average correction period graph.

Assuming that adjacent subsequences are offset by $\mathrm{d}$ points, each subsequence length is L, then the ith sequence can be represented as:

$$
a_{i}(n)=a(n+i d), n=0,1, \cdots, L-1
$$

Among them, the overlapping number of subsequence $a_{i}(n)$ and $a_{i+1}(n)$ is (L-d), if the entire sample point is covered by $K$ subsequences, the relationship between the sample point lengths $\mathrm{N}$ and $\mathrm{K}$ can be expressed as:

$$
N=L+d(K-1)
$$

When using Welch PSD estimation, it's always need to window the subsequence signals. The window function is $w(n)$, the Welch spectral estimate of the signal $a(n)$ can be expressed as:

$$
\hat{P}_{a a}\left(e^{j \omega}\right)=\frac{1}{K L} \sum_{i=0}^{K-1}\left|\sum_{n=0}^{L-1} w(n) a(n+i d) e^{-j n \omega}\right|^{2}
$$

Welch spectral estimation is an asymptotic unbiased estimate of the PSD.

The optimal low-frequency cut-off frequency can be effectively obtained through PSD estimation in acceleration integrals, combined with the frequency domain integration algorithm to obtain the optimal displacement result. By constructing a vibration test stand and designing the actual test of the road surface, the PSD estimation method is used to achieve the estimation of the optimal low-frequency cut-off frequency, and the integration of the acceleration and the integral quality evaluation are realized.

\subsection{Integration Algorithm}

The high-frequency noise of the acceleration signal can be effectively removed by preprocessing such as DC removal and filtering. However, for low-frequency noise, careful consideration is needed. The selection of the cutoff frequency determines the size of the truncation error and will affect the accuracy of the integration. When the vehicle stops moving, the power spectrum intensity of the acceleration signal decreases as the frequency increases. When the vehicle is moving, peaks will appear 
in the power spectrum, and the frequency band where the spikes are located is the frequency band where the acceleration signal is located. Therefore, the most frequent cutoff frequency can be obtained by analyzing the power spectrum of the acceleration signal. The processing flow chart is shown in Fig.1.

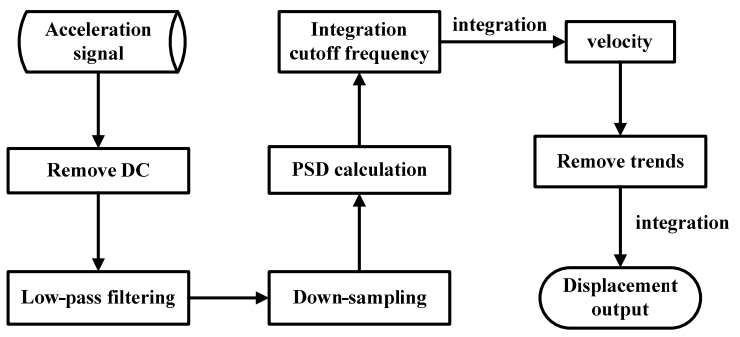

Fig.1. Acceleration processing method flow chart.

The operating steps of acceleration processing method is: (1) Input the dynamic acceleration signal $\mathrm{a}_{0}(\mathrm{t})$, Then after removing the DC component, low-pass filtering, de-noising, down-sampling and other preprocessing, it can get the preconditioned acceleration signal $a(t)$;

(2)By analyzing the PSD sequence $X(k), k=1,2,3, \cdots$ of the pre-processed acceleration signal, record sequence maximum extreme point $k$, commend $k k=k-i+1, m=X(k k-1)-X(k k)$; if $m>$ 0 , then $F_{\text {opt }}=k k^{*} d f$ is the low frequency cutoff frequency that we needed, otherwise, continue to iterate until $\mathrm{m}<0$, then the low frequency cutoff frequency $F_{o p t}$ is obtained by the proposed method. The Algorithm processing flow chart is shown in Fig.2. Where $d f=f_{S} / n f f t$, fs is the sampling frequency, and nfft is the number of points in the Fourier transform.

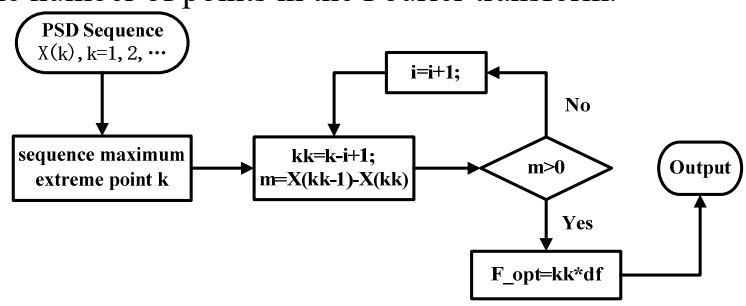

Fig.2. Algorithm flow chart.

(3) The extracted low frequency cutoff frequency is applied to the integral process of the acceleration frequency domain, and the speed signal is obtained by once integration and the displacement signal is obtained by double integration. And use inverse Fourier transform to change them into time domain signals.

(4) Output the displacement signal after the baseline offset correction.

\section{EXPERIMENT AND RESULTS}

In order to verify the effectiveness of the integration algorithm based on PSD estimation, the displacementtime signal and acceleration signal were obtained using a vibration experiment device. Using this method to integrate the acceleration signal, the displacement-time curve obtained by the integration is compared with the displacement-time signal measured by the laser range finder. Because the transient signal is more complex, so the vibration algorithm can be used to evaluate the integral algorithm.

\subsection{Platform And Experiment}

The vibration test bench is shown in Fig.3. The laser displacement sensor and acceleration sensor are installed in the middle of the beam. A detection platform is placed below the beam and its flatness is $0.05 \mathrm{~mm} / 150 \mathrm{~mm}$.

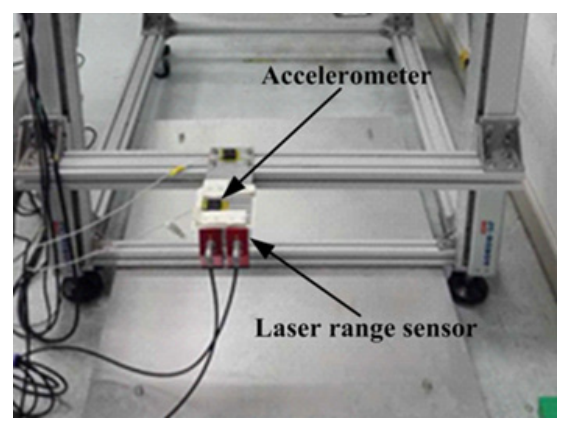

Fig.3. Vibration test bench.

The test bench controls the cross beam by stepping motor to perform vertical reciprocating motion, where the motion amplitude is $6 \mathrm{~mm}$ and the motion speed is about $4 \mathrm{~mm} / \mathrm{s}$. The displacement of the OMS- 8198 laser ranging sensor was used in the experiment with sample rate of $50 \mathrm{kHz}$ and ranging accuracy of $0.05 \mathrm{~mm}$. And SDI 2220-002 uniaxial acceleration sensor was used in the experiment, with response frequency of $400 \mathrm{~Hz}$.

The pre-processed acceleration signal is shown in Fig.4(a). The PSD analysis of the pre-processed acceleration signal is shown in Fig.4(b). From the Fig.4(b), it can be concluded that the extracted low frequency cutoff frequency $(\mathrm{LFCF})$ is $0.2975 \mathrm{~Hz}$. The velocity signal obtained by integrating acceleration once without baseline offset correction and the displacement signal obtained by the second integral are shown in Fig.5(a), (b).It can be seen that there are obvious trend items .The velocity signal obtained by integrating acceleration once with baseline offset correction and the displacement signal obtained by the second integral are shown in Fig.6(a), (b). The schematic diagram of the comparison of the displacement obtained by this method and the measured displacement is shown in Fig.e7(a). The difference between them is shown in Fig.7(b). As can be seen from the Fig.7, the difference between the integral and measured displacement after the PSD processing is $\pm 0.2 \mathrm{~mm}$, which is less than $1 \mathrm{~mm}$ required by the national road test specification.

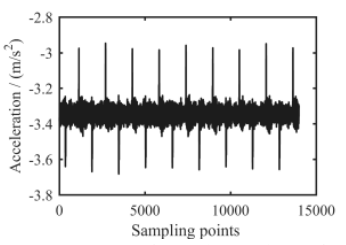

(a) Acceleration signal

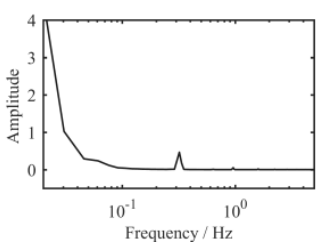

(b) Power spectral density
Fig.4. Acceleration signal and power spectral density. 


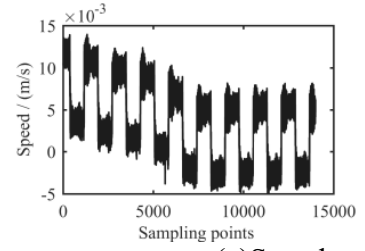

(a)Speed

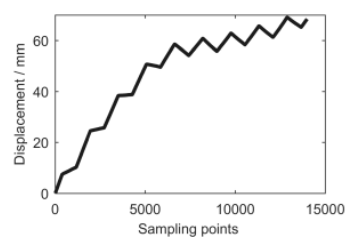

(b) Displacement
Fig.5. Speed and displacement without baseline offset correction.



(a)Speed

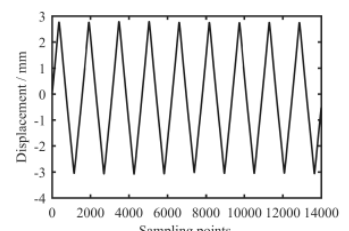

(b) Displacement
Fig.6. Speed and displacement with baseline offset correction.

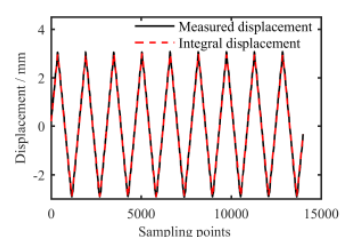

(a) Integral displacement

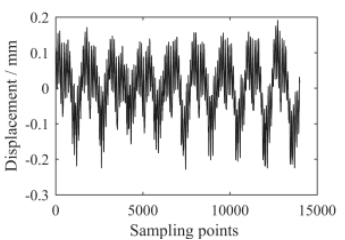

(b) Error curve
Fig.7. Comparion of integral displacement and measured displacement.

\subsection{Displacement Integration Results of Different Cutoff Frequency}

Different low frequency cutoff frequencies (LFCF) will affect the accuracy of the acceleration integral displacement. The optimal low-frequency cut-off frequency (F_opt) is obtained by estimating the power spectrum of the acceleration signal, and the reference low-frequency cut-off frequencies of $0.1 \mathrm{~Hz}$ and $0.32 \mathrm{~Hz}$ are selected at the same time. The results of the integral displacement at different cut-off frequencies are shown in Fig.8(a). The integral displacement error curve is shown in Fig.8(b).

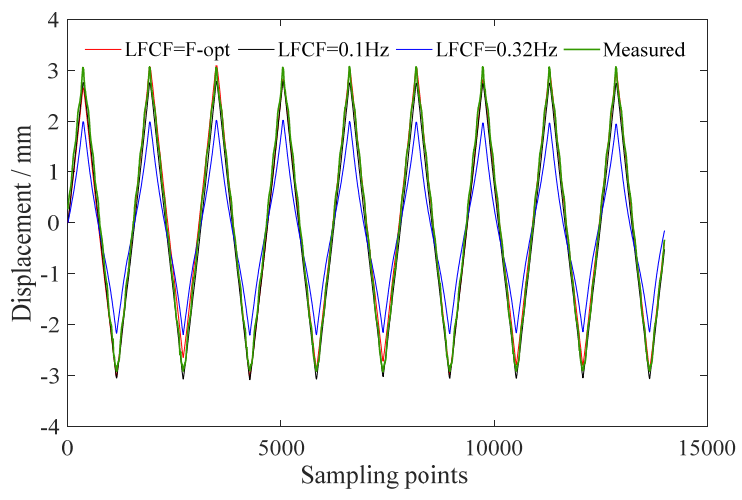

(a) Integrated displacement Curves of different LFDF

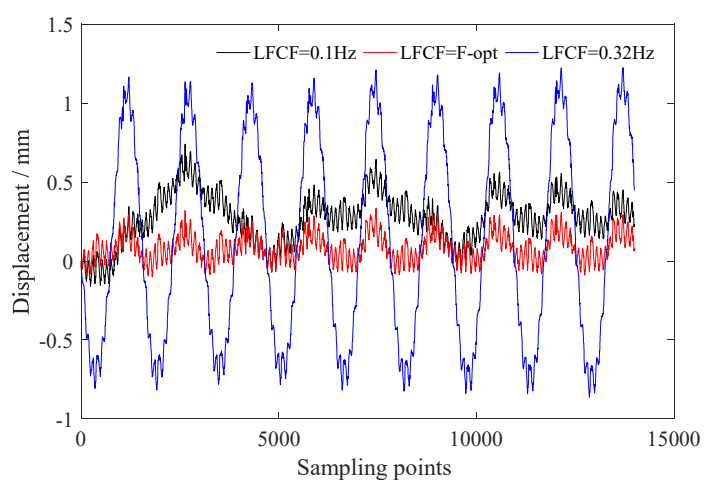

(b) Error curves of different LFCF

Fig.8. Comparison of different low frequency cut-off frequency.

As can be seen from Figure8, when the integrated low-frequency cut-off frequency LFCF $=0.1 \mathrm{~Hz}$, the integral error is about $0.6 \mathrm{~mm}$. When the integral cut-off frequency $\mathrm{LFCF}=0.32 \mathrm{~Hz}$, the integration error reaches $1.3 \mathrm{~mm}$. It can be seen in the figure that the integration error increases when the selected low frequency cut-off frequency is higher or lower than the optimal cutoff frequency $\left(F_{-}\right.$opt). The test shows that the effectiveness of the proposed algorithm is verified.

\section{CONCLUSIONS}

Acquiring the displacement by integrating the acceleration signal twice is widely used in the aspects of carrier vibration testing and displacement detection. It is of great significance to improve the integral accuracy of acceleration. Based on the traditional frequency-domain integration, this paper proposed a novel method to obtain the best integration cut-off frequency by estimating the power spectrum of the acceleration, thereby improving the integration accuracy. Through the detection of the vibration test platform, it is proved that the algorithm can effectively intercept the integration frequency band and achieve the optimization of the integration result, so that the integration error is controlled within $\pm 0.2 \mathrm{~mm}$. The next step will further optimize the algorithm and complete the actual road surface application test to achieve the purpose of engineering application.

\section{REFERENCES}

1. Q Xu. Journal of Vibration Measurement \& Diagnosis, (1997).

2. N Li, DS Li, HN Li. Scientia Sinica, (2016).

3. F Cai. Chinese Journal of Scientific Instrument, (2006).

4. A Smyth, ML Wu. Mechanical Systems and Signal Processing,21: 706-723(2007) .

5. MK Gu, ZH Lv. Mechanical Science \& Technology for Aerospace Engineering, (2011).

6. JF Wang.computer science, 37 :201-202, 237(2010).

7. YH Hong, HK Kim ,HS Lee .Journal of Sound \& 
Vibration, 329(23):4980-5003(2010).

8. HS Lee, YH Hong, HW Park . International

Journal for Numerical Methods in Engineering, 82(4):403-434(2010).

9. B Lotfi ,L Huang .Measurement, 90:242-249(2016). 